\title{
ERRATUM TO "LEVEL-RANK DUALITY FOR VERTEX OPERATOR ALGEBRAS OF TYPES $B$ AND $D$ "
}

\author{
CUIPO JIANG ${ }^{1, a}$ AND CHING HUNG LAM ${ }^{2, b}$
}

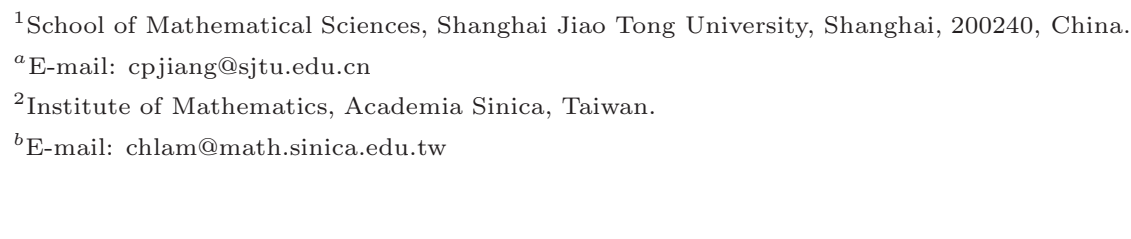

\section{Erratum}

This is an erratum to the article "Level-rank duality for vertex operator algebras of types $B$ and $D "[3]$.

In [3], we determined the commutant vertex subalgebra $C_{L_{\widehat{\mathfrak{s}} m}(1,0) \otimes n}\left(L_{\widehat{\mathfrak{s o}}_{m}}(n, 0)\right)$ (cf. Theorems 4.13, 4.15 and 4.17). While the main result holds for $m, n \geq 4$, it is not correct when $n=3$. The correct statement should be amended as follows:

(1) For $m, n \geq 4$,

$$
C_{L_{\widehat{\mathfrak{s o}} m}(1,0) \otimes n}\left(L_{\widehat{\mathfrak{s o}}_{m}}(n, 0)\right)=L_{\widehat{\mathfrak{s o}}_{n}}(m, 0)^{G}
$$

if $m$ or $n$ is odd and

$$
C_{L_{\widehat{\mathfrak{s}}_{m}}(1,0)^{\otimes n}}\left(L_{\widehat{\mathfrak{s o}}_{m}}(n, 0)\right)=\left(L_{\widehat{\mathfrak{s o}}_{n}}(m, 0) \oplus L_{\widehat{\mathfrak{s}}_{n}}\left(m, m \Lambda_{1}\right)\right)^{G}
$$

if both $m, n$ are even.

(2) For $m \geq 4$ and $n=3$,

$$
C_{L_{\widehat{\mathfrak{s}} m}(1,0)^{\otimes 3}}\left(L_{\widehat{\mathfrak{s o}} m}(3,0)\right)=\left(L_{\widehat{\mathfrak{s l}}}(2 m, 0)+L_{\widehat{\mathfrak{s}} \widehat{I}_{2}}(2 m, 2 m)\right)^{G}
$$

AMS Subject Classification: 17B69.

Key words and phrases: Level rank duality, vertex operator algebras, affine Lie algebras. 
if $m$ is even and

$$
C_{L_{\widehat{\mathfrak{s o}} m}(1,0)^{\otimes 3}}\left(L_{\widehat{\mathfrak{s o}} m}(3,0)\right)=L_{\widehat{\mathfrak{s l}}}(2 m, 0)^{G}
$$

if $m$ is odd.

Here $G$ is an abelian subgroup of Aut $\left(L_{\widehat{s o}_{m n}}(1,0)\right)$ such that $L_{\widehat{s o} m n}(1,0)^{G}$ is isomorphic to the tensor product VOA $L_{\widehat{s o m}}(1,0)^{\otimes n}$ (see [3, p.44] for detail).

\subsection{Fermionic vertex superalgebras}

As in [3], we consider the Clifford algebra $\mathcal{C} l_{2 m}$ generated by $\psi_{i}^{ \pm}(r), 1 \leq$ $i \leq m, r \in \mathbb{Z}+\frac{1}{2}$, satisfying the non-trivial relations

$$
\left[\psi_{i}^{ \pm}(r), \psi_{j}^{\mp}(s)\right]_{+}=\psi_{i}^{ \pm}(r) \psi_{j}^{\mp}(s)+\psi_{j}^{\mp}(s) \psi_{i}^{ \pm}(r)=\delta_{r+s, 0} \delta_{i j},
$$

where $m \in \mathbb{Z}_{+}, 1 \leq i, j \leq m$, and $r, s \in \mathbb{Z}+\frac{1}{2}$.

We also use $\mathcal{C} l_{2 m+1}$ to denote the Clifford algebra generated by

$$
\psi_{i}^{ \pm}(r), \psi_{2 m+1}(r), r \in \mathbb{Z}+\frac{1}{2}, 1 \leq i \leq m
$$

with the non-trivial relations

$$
\begin{aligned}
{\left[\psi_{i}^{ \pm}(r), \psi_{k}^{\mp}(s)\right]_{+} } & =\delta_{i k} \delta_{r+s, 0}, \\
{\left[\psi_{2 m+1}(r), \psi_{2 m+1}(s)\right]_{+} } & =\delta_{r+s, 0},
\end{aligned}
$$

where $1 \leq i, k \leq m, r, s \in \mathbb{Z}+\frac{1}{2}$.

Let $\mathcal{F}_{2 m}$ be the irreducible $\mathcal{C l}_{2 m}$-module generated by the cyclic vector 1 such that

$$
\psi_{i}^{ \pm}(r) \mathbf{1}=0, \text { for } r>0,1 \leq i \leq m
$$

and $\mathcal{F}_{2 m+1}$ the irreducible $\mathcal{C l}_{2 m+1}$-module generated by the cyclic vector $\mathbf{1}$ such that

$$
\psi_{i}^{ \pm}(r) \mathbf{1}=\psi_{2 m+1}(r) \mathbf{1}=0, \text { for } r>0,1 \leq i \leq m .
$$


It is well-known [1, 2] that both $\mathcal{F}_{2 m}$ and $\mathcal{F}_{2 m+1}$ are vertex operator super algebras.

The main idea of [3] is to identify the even part $\left(\mathcal{F}_{m}\right)^{\text {even }}$ with the affine VOA $L_{\widehat{\mathfrak{S o}} m}(1,0)$ (cf. [3, Theorems 3.1 and 3.2]); however, the identification stated in [3] is not correct when $m$ is small. The correct statement is as follows.

Theorem 1.1. For $m \in \mathbb{Z}_{\geq 4}$, we have

$$
\left(\mathcal{F}_{m}\right)^{\text {even }} \cong L_{\widehat{\mathfrak{s o}}_{m}}(1,0)
$$

Moreover,

$$
\left(\mathcal{F}_{3}\right)^{\text {even }} \cong L_{\widehat{\mathfrak{s l}}_{2}}(2,0) \quad \text { and } \quad\left(\mathcal{F}_{2}\right)^{\text {even }} \cong V_{\sqrt{2} A_{1}}
$$

Therefore, the analysis in [3] is still valid when $m, n \geq 4$ but the cases for $n=2$ and $n=3$ require special treatments. In [3] , the case for $n=2$ was also studied (see [3, Sections 4.1.1 and 4.2.1]. In the following, we will provide the argument for the case $n=3$.

\subsection{The case $n=3$ and $m \in \mathbb{Z}_{\geq 4}$}

By using Table 3 of [4] and the fact that $\mathcal{F}_{3}^{\text {even }}$ is isomorphic to $L_{\widehat{\mathfrak{s l}_{2}}}(2,0)$, we have

$$
L_{\widehat{\mathfrak{s}}_{3 m}}(1,0) \supset L_{\widehat{\mathfrak{s}}_{m}}(3,0) \otimes L_{\widehat{\mathfrak{s l}}_{2}}(2 m, 0)
$$

as a full subVOA.

For the even cases, we relabel the generators of the Clifford algebra $\mathcal{C} l_{6 m}$ so that it is generated by $\psi_{i j}^{ \pm}(r), 1 \leq i \leq m, 1 \leq j \leq 3, r \in \mathbb{Z}+\frac{1}{2}$, with the non-trivial relations

$$
\left[\psi_{i j}^{ \pm}(r), \psi_{k l}^{\mp}(s)\right]_{+}=\delta_{i k} \delta_{j l} \delta_{r+s, 0},
$$

where $1 \leq i, k \leq m, 1 \leq j, l \leq 3, r, s \in \mathbb{Z}+\frac{1}{2}$. We also set

$$
\begin{aligned}
\psi_{k j}\left(-\frac{1}{2}\right) \mathbf{1} & =\frac{1}{\sqrt{2}}\left(\psi_{k j}^{+}\left(-\frac{1}{2}\right) \mathbf{1}+\psi_{k j}^{-}\left(-\frac{1}{2}\right) \mathbf{1}\right), \\
\psi_{m+k, j}\left(-\frac{1}{2}\right) \mathbf{1} & =\frac{-\sqrt{-1}}{\sqrt{2}}\left(\psi_{k j}^{+}\left(-\frac{1}{2}\right) \mathbf{1}-\psi_{k j}^{-}\left(-\frac{1}{2}\right) \mathbf{1}\right),
\end{aligned}
$$


for $1 \leq k \leq m, 1 \leq j \leq 3$. In this case, $C_{L_{\widehat{\mathfrak{s o}}_{6 m}}(1,0)}\left(L_{\widehat{\mathfrak{s o}}_{2 m}}(3,0)\right)$ contains a highest weight vector

$$
u^{(12)}=\prod_{k=1}^{2 m}\left(\psi_{k 1}\left(-\frac{1}{2}\right)+\sqrt{-1} \psi_{k 2}\left(-\frac{1}{2}\right)\right) \mathbf{1}
$$

and it generates the $L_{\widehat{\mathfrak{s L}_{2}}}(4 k, 0)$-module $L_{\widehat{\mathfrak{s l}_{2}}}(4 k, 4 k)$. Hence, we have the following result.

Lemma 1.2. For $m \in \mathbb{Z}_{\geq 2}$, we have

$$
C_{L_{\widehat{\mathfrak{s o}}_{6 m}}(1,0)}\left(L_{\widehat{\mathfrak{s o}}_{2 m}}(3,0)\right)=L_{\widehat{\mathfrak{s l}_{2}}}(4 m, 0)+L_{\widehat{\mathfrak{s l}_{2}}}(4 m, 4 m) .
$$

By the definition of $G$, we also have the following result.

Theorem 1.3. For $m \in \mathbb{Z}_{\geq 2}$,

$$
C_{L_{\widehat{\mathfrak{s}}_{2}}(1,0) \otimes 3}\left(L_{\widehat{\mathfrak{s o}}_{2 m}}(3,0)\right)=\left(L_{\widehat{\mathfrak{s l}_{2}}}(4 m, 0)+L_{\widehat{\mathfrak{s l}_{2}}}(4 m, 4 m)\right)^{G} .
$$

Similarly, by using Table 3 of [4], we can deduce that for $m \geq 2$,

$$
C_{L_{\widehat{\mathfrak{s}}_{3(2 m+1)}}(1,0)}\left(L_{\widehat{\mathfrak{s}}_{2} 2 m+1}(3,0)\right)=L_{\widehat{\mathfrak{s l}}_{2}}(4 m+2,0) .
$$

Thus, we have the following result.

Theorem 1.4. For $m \geq 2$, we have

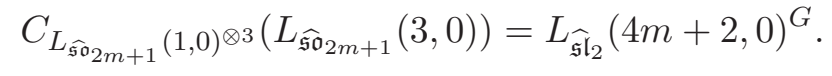

\section{References}

1. A. J. Feingold, I. B. Frenkel, Classical affine Lie algebras, Adv. Math., 56 (1985), 117-172.

2. A. J. Feingold, I. B. Frenkel, J. Ries, Spinor construction of vertex operator algebras, Triality, and $E_{8}^{(1)}$, Amer. Math. Soc., Providence, 1991.

3. C. Jiang and C. H Lam, Level-rank duality for vertex operator algebras of type B and D, Bull. Inst. Math. Acad. Sin. (N.S.) 14 (2019), no. 1, 31-54.

4. V. G. Kac, Pierluigi Möseneder Frajria, Paolo Papi, and Feng Xu, Conformal embedding and simple current extensions, Int. Math. Res. Not. 2015, no. 14, 5229-5288; arXiv: $1210.6602 \mathrm{v} 1$. 